\title{
The dark side of clock-controlled flowering
}

\section{Vicente Rubio $^{1 *}$ and Xing Wang Deng ${ }^{2}$}

\begin{abstract}
Addresses: ${ }^{1}$ Department of Plant Molecular Genetics, Centro Nacional de Biotecnología-CSIC, Darwin 3, Campus UAM, Cantoblanco, Madrid, 28049, Spain; ${ }^{2}$ Department of Molecular, Cellular, and Developmental Biology, Yale University, 165 Prospect Street, OML 352A, New Haven, CT 06520, USA

*Corresponding author: Vicente Rubio (vrubio@cnb.csic.es)

FI000 Biology Reports 2009, I:57 (doi:10.34I0/BI-57)

The electronic version of this article is the complete one and can be found at: http://FI000.com/Reports/Biology/content/I/57

Abstract

Perception of seasonal changes in day length allows plants to properly maintain daily biological rhythms and determine the most favorable time for flowering. Important knowledge has been gained recently on the molecular basis of this process, which depends not only on light perception at certain times of day but also on its dark phase.
\end{abstract}

\section{Introduction and context}

As in many other organisms, plants rely on circadian clocks, endogenous self-sustained molecular oscillators, to keep track of time and to generate biological rhythms that occur on a daily basis [1]. Additionally, clock function allows flowering plants to estimate the most appropriate time of year to flower, thus favoring their reproductive success. For this, the plant clock needs to be reset (or synchronized) to the external day/night cycles. This process implies the perception of fluctuations in day length (photoperiod) and temperature, and subsequent transmission of such environmental information through specific 'input pathways' to the clock. Three major classes of photoreceptors are known to mediate light-input signaling to the clock in the model plant Arabidopsis: blue light (BL) receptors of the ZEITLUPE (ZTL) and cryptochrome (CRY) families and the red/farred light-sensing phytochromes (Phys) [2]. Studies over the past two years have shed light on the molecular mechanisms that regulate their function in controlling clock resetting by light (CRL) and photoperiodic flowering. These mechanisms largely involve CONSTITUTIVE PHOTOMORPHOGENESIS 1 (COP1), a master repressor of light-mediated development in darkness.

\section{Major recent advances}

Members of the ZTL/FKF1 (FLAVIN-BINDING, KELCH REPEAT, F-BOX 1)/LKP2 (LOV, KELCH PROTEIN 2) family of photoreceptors display two signature motifs: a BL-sensing LOV domain and an F-box motif involved in ubiquitination and degradation of specific protein substrates [3]. Indeed, both domains are essential for ZTL and FKF1 control of CRL and photoperiodic flowering, respectively. Thus, upon BL perception, ZTL and FKF1 form stable complexes with GIGANTEA (GI), a clockregulatory protein [4]. BL-enhanced ZTL-GI interaction enables ZTL stabilization during the late evening, thus favoring degradation of the ZTL targets, such as TIMING OF CAB1 (TOC1) and PSEUDORESPONSE REGULATOR 5 (PRR5) [4,5] (Figure 1). TOC1 and PRR5 are clock oscillator components whose rhythmic accumulation is necessary to drive robust oscillations of clock-generated biological rhythms. Therefore, day-length information can be transmitted to the clock through ZTL-GI action on TOC1 and PRR5 to influence timing and amplitude of circadian rhythms. FKF1-GI complexes also accumulate during the evening to effectively promote degradation of CYCLING DOF FACTOR 1 (CDF1) [6]. CDF1 negatively controls flowering by repressing expression of the floral inducer gene CONSTANS (CO) [7]. Therefore, daylength-dependent formation of FKF1-GI-CDF1 complexes allows precise timing of $\mathrm{CO}$ expression, thus helping to determine the most advantageous time for flowering.

Recently, we reported that COP1, an E3 ubiquitin ligase that promotes degradation of positive regulators of 
Figure I. Photoreceptor-mediated signaling pathways converge to allow clock resetting by light and photoperiodic flowering

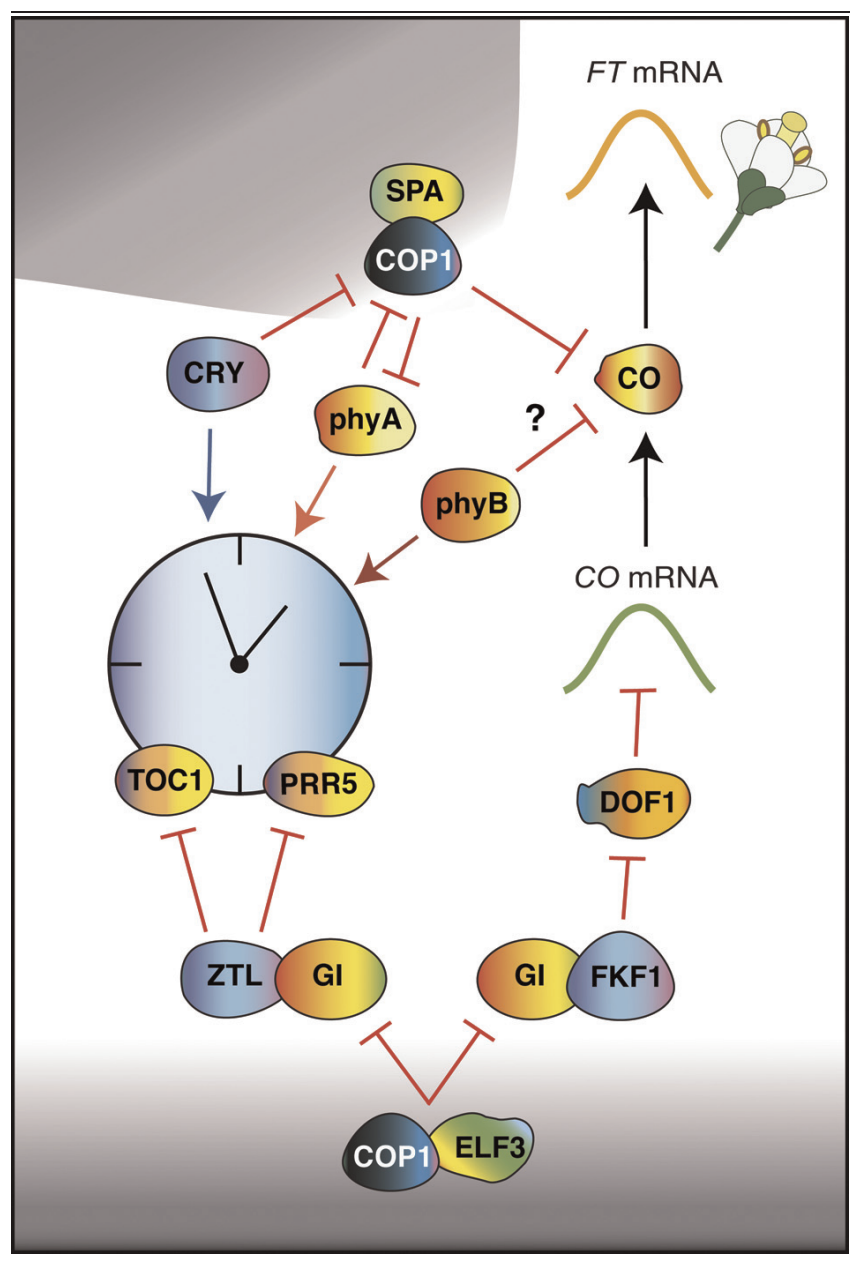

The molecular mechanisms by which these pathways exert their function significantly involve control of the accumulation of limiting regulatory proteins that modulate circadian oscillation and/or the promotion of flowering (for example, TOCI, PRR5, DOFI, or CO). COPI plays a central role in reducing the effectiveness of these light-input pathways. For this, COPI, likely with the help of substrate adaptors (for example, ELF3 and SPA proteins), triggers dark-driven degradation of regulators of clock function and/or flowering, such as GI, CO, and PhyA. CDFI, CYCLING DOF FACTOR I; CO, CONSTANS; COPI, CONSTITUTIVE PHOTOMORPHOGENESIS I; CRY, cryptochrome; ELF3, EARLY-FLOWERING 3; FKFI, FLAVIN-BINDING, KELCH REPEAT, F-BOX I; FT, FLOWERING LOCUS T; GI, GIGANTEA; Phy, phytochrome; PRR5, PSEUDORESPONSE REGULATOR 5; SPA, SUPPRESSOR OF PHYA-105; TOCI, TIMING OF CABI; ZTL, ZEITLUPE.

photomorphogenesis (light-mediated development) in darkness, also limits the accumulation of GI [8] (Figure 1). This process is enabled by EARLY-FLOWERING 3 (ELF3), a repressor of CRL and flowering which probably acts as a substrate adaptor bringing together COP1 and GI. By reducing GI protein levels at night,
COP1 and ELF3 may reduce the abundance of the ZTLand FKF1-GI complexes, desensitizing the clock to light signals after dusk and repressing photoperiodic flowering. An interesting additional finding was that ELF3 is degraded upon interaction with COP1, suggesting that a negative-feedback mechanism limits the extent of ELF3 activity to the night phase. This inhibitory mechanism may help to restrict clock responsiveness to light to certain times of day, a process known as circadian gating of light.

Two recent studies have shown that $\mathrm{CO}$ protein is also a target of COP1 E3 ubiquitin-ligase activity $[9,10]$ (Figure 1). Therefore, COP1 control of CO function may be accomplished by two separate mechanisms: transcriptional regulation of the CO gene, by controlling FKF1-GI activity, and post-translational control of $\mathrm{CO}$ accumulation, by promoting its proteasomal degradation in darkness [8-11]. Both regulatory mechanisms may be repressed by light-activated CRYs, which is in agreement with previous results showing that CRYs physically interact with COP1 and impair its function under BL conditions $[12,13]$. Besides controlling $\mathrm{CO}$ and, potentially, GI accumulation, a direct mechanism by which photoactive CRYs regulate flowering gene expression was recently unveiled [14]. It has been found that CRY2 physically interacts with transcription factor (TF) CRY-INTERACTING BASIC-HELIX-LOOP-HELIX (CIB1) to promote expression of FLOWERING LOCUS T (FT), a gene that encodes a flowering-inducing mobile protein: the florigen [15-18]. Whether COP1 controls CRY2-mediated activation of CIB1 remains to be demonstrated.

Concerning Phys, there is evidence that PhyA and PhyB differentially control CO stability, with PhyA stabilizing $\mathrm{CO}$ in the evening and PhyB promoting its degradation at dawn $[9,11]$. These two receptors are also known to transmit light information to the clock [19]. However, contrary to the case of ZTL, FKF1, and CRYs, the molecular mechanisms by which Phys affect CRL and flowering are poorly understood. One possibility is that Phys act in concert with CRYs to modulate COP1 function toward GI or $\mathrm{CO}$ proteins. This may be supported by the fact that PhyA, which is known to interact with CRY1, transmits both low-fluence red light and BL to the clock $[19,20]$. Functional relationships between CRYs and Phys are also supported by physical interaction of CRY2 with PhyB [21]. Another possibility is that, as in the case of CRY2, Phys directly affect the function of TFs controlling circadian and flowering gene expression. In agreement with this, PhyA and PhyB bind PHY-INTERACTING FACTOR 3 (PIF3), a TF that negatively regulates $C O$ and FT gene expression [22]. 
Similarly, it has been shown that FAR-RED ELONGATED HYPOCOTYL 3 (FHY3), a TF that mediates Phys signaling, also participates in the gating of red light signals for clock resetting [23]. FHY3 association with underphosphorylated PhyA was recently found to protect PhyA from targeted degradation by COP1, thus adding a new layer of regulation to COP1-mediated control of photoreceptors [24].

\section{Future directions}

How COP1 can recognize diverse unrelated protein targets, such as PhyA, CO, or GI, represents an important question arising from these studies. The fact that ELF3 likely functions as a substrate adaptor for COP1 to recognize GI may answer this question [8]. Thus, COP1 may interact with additional specific adaptors to discriminate between targets depending on precise developmental stages or light-controlled biological processes. In this regard, it was shown that COP1 is part of a multi-protein complex that contains, among others, members of the SUPPRESSOR OF PHYA-105 (SPA) protein family [25] (Figure 1). SPA proteins share sequence similarity with COP1 and modulate its E3 ubiquitin-ligase activity toward light-response regulators such as ELONGATED HYPOCOTYL 5 (HY5), LONG AFTER FAR-RED 1 (LAF1), and PhyA [25-29]. This function is likely extended to other substrates, such as CO [27]. It would be interesting to analyze whether different combinations of COP1, SPA members, and additional proteins, like ELF3, determine target specificity of COP1.

Despite important insights, our knowledge about the regulatory mechanisms governing CRL and photoperiodic flowering is still very limited. Thus, studies in this field very often reveal new genetic and molecular interactions as well as new regulatory activities (for example, phosphorylation, sumoylation, and histone acetylation) within these processes. These findings should allow us to further explore how light/dark signaling pathways converge to control light resetting of the clock and flowering time.

\section{Abbreviations}

$\mathrm{BL}$, blue light; CDF1, CYCLING DOF FACTOR 1; CIB1, CRYPTOCHROME-INTERACTING BASIC-HELIXLOOP-HELIX 1; CO, CONSTANS; COP1, CONSTITUTIVE PHOTOMORPHOGENESIS 1; CRL, clock-resetting by light; CRY, cryptochrome; ELF3, EARLY-FLOWERING 3; FHY3, FAR-RED ELONGATED HYPOCOTYL 3; FKF1, FLAVIN-BINDING, KELCH REPEAT, F-BOX 1; FT, FLOWERING LOCUS T; GI, GIGANTEA; HY5, ELONGATED HYPOCOTYL 5; LAF1, LONG AFTER FAR-RED 1; LKP2, LOV, KELCH PROTEIN 2; Phy, phytochrome; PIF3, PHYINTERACTING FACTOR 3; PRR5, PSEUDORESPONSE
REGULATOR 5; SPA, SUPPRESSOR OF PHYA-105; TF, transcription factor; TOC1, TIMING OF CAB1; ZTL, ZEITLUPE.

\section{Competing interests}

The authors declare that they have no competing interests.

\section{Acknowledgments}

We would like to apologize to all researchers whose work we were not able to mention due to space constraints. We are grateful to Roberto Solano for critical reading of the manuscript. VR is supported by the Spanish Ministry of Science and Innovation (MICINN) under the 'Ramón y Cajal' Program and by grants S-GEN/0191/2006 (from the Comunidad de Madrid) and EUI2008-03742 (MICINN; Plant-KBBE Program). The relevant research in the lab of XWD was supported by the National Institutes of Health (NIH; grant GM-47850).

\section{References}

I. Más P: Circadian clock function in Arabidopsis thaliana: time beyond transcription. Trends Cell Biol 2008, 18:273-8I.

2. Jiao $Y$, Lau OS, Deng XW: Light-regulated transcriptional networks in higher plants. Nat Rev Genet 2007, 8:217-30.

3. Somers DE, Fujiwara S: Thinking outside the F-box: novel ligands for novel receptors. Trends Plant Sci 2009, I4:206-I3.

4. Kim WY, Fujiwara S, Suh SS, Kim J, Kim Y, Han L, David K, Putterill J, Nam HG, Somers DE: ZEITLUPE is a circadian photoreceptor stabilized by GIGANTEA in blue light. Nature 2007, 449:356-60.

FI000 Factor 8.2 Exceptional

Evaluated by C Robertson McClung 05 Oct 2007, Julin Maloof 09 Oct 2007, Judy Callis 18 Oct 2007

5. Fujiwara S, Wang L, Han L, Suh SS, Salomé PA, McClung CR, Somers DE: Post-translational regulation of the Arabidopsis circadian clock through selective proteolysis and phosphorylation of pseudo-response regulator proteins. J Biol Chem 2008, 283:23073-83.

FI000 Factor 3.0 Recommended

Evaluated by Judy Callis OI Aug 2008

6. Sawa M, Nusinow DA, Kay SA, Imaizumi T: FKFI and GIGANTEA complex formation is required for day-length measurement in Arabidopsis. Science 2007, 3 1 8:26I-5.

7. Imaizumi T, Schultz TF, Harmon FG, Ho LA, Kay SA: FKF I F-box protein mediates cyclic degradation of a repressor of CONSTANS in Arabidopsis. Science 2005, 309:293-7.

FI000 Factor 4.9 Must Read

Evaluated by Tai-ping Sun 26 Jul 2005, Richard M Amasino 26 Jul 2005, Judy Callis 03 Aug 2005

8. Yu JW, Rubio V, Lee NY, Bai S, Lee SY, Kim SS, Liu L, Zhang Y, Irigoyen ML, Sullivan JA, Zhang Y, Lee I, Xie Q, Paek NC, Deng XW: COPI and ELF3 control circadian function and photoperiodic flowering by regulating GI stability. Mol Cell 2008, 32:617-30.

9. Jang S, Marchal V, Panigrahi KC, Wenkel S, Soppe W, Deng XW, Valverde F, Coupland G: Arabidopsis COPI shapes the temporal pattern of $\mathrm{CO}$ accumulation conferring a photoperiodic flowering response. EMBO J 2008, 27:1277-88.

10. Liu LJ, Zhang YC, Li QH, Sang Y, Mao J, Lian HL, Wang L, Yang HQ: COPI-mediated ubiquitination of CONSTANS is implicated 
in cryptochrome regulation of flowering in Arabidopsis. Plant Cell 2008, 20:292-306.

II. Valverde F, Mouradov A, Soppe W, Ravenscroft D, Samach A, Coupland G: Photoreceptor regulation of CONSTANS protein in photoperiodic flowering. Science 2004, 303:1003-6.

FI000 Factor 8.0 Exceptional

Evaluated by Michael Lenhard 25 Feb 2004, Richard M Amasino 02 Apr 2004

12. Wang H, Ma LG, Li JM, Zhao HY, Deng XW: Direct interaction of Arabidopsis cryptochromes with COPI in light control development. Science 200I, 294:154-8.

FI000 Factor 6.0 Must Read

Evaluated by Judy Callis 28 Nov 200 I

13. Yang HQ, Tang RH, Cashmore AR: The signaling mechanism of Arabidopsis CRYI involves direct interaction with COPI. Plant Cell 200I, I3:2573-87.

14. Liu H, Yu X, Li K, Klejnot J, Yang H, Lisiero D, Lin C: Photoexcited CRY2 interacts with CIBI to regulate transcription and floral initiation in Arabidopsis. Science 2008, 322:1535-9.

FI000 Factor 3.0 Recommended

Evaluated by Xing Wang Deng 09 Dec 2008

15. Corbesier L, Vincent C, Jang S, Fornara F, Fan Q, Searle I, Giakountis A, Farrona S, Gissot L, Turnbull C, Coupland G: FT protein movement contributes to long-distance signaling in floral induction of Arabidopsis. Science 2007, 316:1030-3.

FI000 Factor 3.0 Recommended

Evaluated by Daniel Gallie 13 Jun 2007

16. Jaeger KE, Wigge PA: FT protein acts as a long-range signal in Arabidopsis. Curr Biol 2007, 17:1050-4.

FI000 Factor 3.0 Recommended

Evaluated by Julia Kehr 08 Jun 2007

17. Mathieu J, Warthmann N, Küttner F, Schmid M: Export of FT protein from phloem companion cells is sufficient for floral induction in Arabidopsis. Curr Biol 2007, 17:1055-60.

FI000 Factor 3.0 Recommended Evaluated by Julin Maloof 27 Jul 2007

18. Tamaki S, Matsuo S, Wong HL, Yokoi S, Shimamoto K: Hd3a protein is a mobile flowering signal in rice. Science 2007, 316:1033-6.

FI000 Factor 3.0 Recommended

Evaluated by Daniel Gallie 13 Jun 2007
19. Somers DE, Devlin PF, Kay SA: Phytochromes and cryptochromes in the entrainment of the Arabidopsis circadian clock. Science 1998, 282:1488-90.

20. Ahmad M, Jarillo JA, Smirnova O, Cashmore AR: The CRYI blue light photoreceptor of Arabidopsis interacts with phytochrome A in vitro. Mol Cell 1998, I:939-48.

21. Más P, Devlin PF, Panda S, Kay SA: Functional interaction of phytochrome B and cryptochrome 2. Nature 2000, 408:207-II.

22. Oda A, Fujiwara S, Kamada H, Coupland G, Mizoguchi T: Antisense suppression of the Arabidopsis PIF3 gene does not affect circadian rhythms but causes early flowering and increases FT expression. FEBS Lett 2004, 557:259-64.

23. Allen $T$, Koustenis A, Theodorou G, Somers DE, Kay SA, Whitelam GC, Devlin PF: Arabidopsis FHY3 specifically gates phytochrome signaling to the circadian clock. Plant Cell 2006 , 18:2506-16.

24. Saijo Y, Zhu D, Li J, Rubio V, Zhou Z, Shen Y, Hoecker U, Wang H, Deng XW: Arabidopsis COPI/SPAI complex and FHYI/FHY3 associate with distinct phosphorylated forms of phytochrome A in balancing light signaling. Mol Cell 2008, 31:607-13.

25. Zhu $D$, Maier A, Lee $J H$, Laubinger S, Saijo $Y$, Wang $H$, Ou LJ, Hoecker U, Deng XW: Biochemical characterization of Arabidopsis complexes containing CONSTITUTIVELY PHOTOMORPHOGENICI and SUPPRESSOR OF PHYA proteins in light control of plant development. Plant Cell 2008, 20:2307-23.

26. Saijo $Y$, Sullivan JA, Wang $H$, Yang J, Shen $Y$, Rubio V, Ma L, Hoecker U, Deng XW: The COPI-SPAI interaction defines a critical step in phytochrome A-mediated regulation of HY5 activity. Genes Dev 2003, 17:2642-7.

27. Seo HS, Watanabe E, Tokutomi S, Nagatani A, Chua NH: Photoreceptor ubiquitination by COPI E3 ligase desensitizes phytochrome A signaling. Genes Dev 2004, 18:617-22.

FI000 Factor 4.9 Must Read

Evaluated by Pascal Genschik 29 Mar 2004, Xing Wang Deng 07 Jul 2004, Tai-ping Sun 03 Aug 2004

28. Seo HS, Yang JY, Ishikawa M, Bolle C, Ballesteros ML, Chua NH: LAFI ubiquitination by COPI controls photomorphogenesis and is stimulated by SPAI. Nature 2003, 423:995-9.

FI000 Factor 3.0 Recommended Evaluated by Judy Callis 29 Jul 2003

29. Laubinger S, Marchal V, Le Gourrierec J, Wenkel S, Adrian J, Jang S, Kulajta C, Braun H, Coupland G, Hoecker U: Arabidopsis SPA proteins regulate photoperiodic flowering and interact with the floral inducer CONSTANS to regulate its stability. Development 2006, 133:3213-22. 